\title{
Abnormal Cingulate Modulation of Fronto-Temporal Connectivity in Schizophrenia
}

\author{
P. Fletcher,* P. J . McKenna,† K. J . Friston,* C. D. Frith,* and R. J . Dolan*, \\ *The Wel Icome Department of Cognitive Neurology, I nstitute of Neurol ogy, London WC1N 3BG; †Department of Psychol ogical Medicine, \\ Fulbourn Hospital, Cambridge; and ¥TheRoyal FreeHospital Medical School, Pond Street, London NW3, United Kingdom
}

Received August 17, 1998

Functional neuroimaging provides a novel means of exploring neurophysiological function in schizophrenia. However, most of the studies that have been carried out report their findings in terms of regionally localized abnormalities. In this paper we propose an alternative method of data analysis that emphasizes global integration rather than isolated regional changes in response to psychological tasks. In doing so, we suggest that brain abnormalities in schizophrenia are best characterized as a disturbance in the integration of activity across a number of brain regions. Using a hypothesis-led analysis, we show that the condition is associated with a disruption of the normal anterior cingulate modulation of prefrontotemporal integration. This analytical technique, we suggest, provides a conceptually powerful approach to the imaging of abnormal brain function in psychopathological conditions. 1999 Academic Press

\section{INTRODUCTION}

Increasing sophistication of functional neuroimaging techniques offers a more precise means of describing brain abnormalities in psychiatric conditions such as schizophrenia (Andreasen, 1996). However, these new methods have not, as yet, identified a defining pathophysiology and often produce results that are inconsistent (Chua and McKenna, 1995). In this paper, we suggest that one problem may be a tendency to characterize brain activity purely in terms of anatomically segregated responses. While undoubtedly useful, this approach may have profound limitations when trying to explain complex disorders such as schizophrenia.

Common to the majority of functional neuroimaging studies is a widely held theoretical approach to brain organization, that of functional segregation. This embraces the idea that specific cognitive processes or operations are localized to discrete anatomical modules (Phillips et al., 1984). A characterization of brain function based on the segregationist approach is incomplete insofar as it neglects a description of the relationships between different brain regions. A functional integration approach, on the other hand, considers cognitive processes as emergent properties of interconnected, though anatomically separate, modules (Tononi et al., 1992). Studies of functional integration attempt to characterize the ways in which brain regions interact. Such connectivity has been described in two related but crucially different ways (Friston et al., 1993, 1993). Brain regions $A$ and $B$ may be considered functionally connected if it can be shown that, across time, an increase (or decrease) of activity in region A is associated with an increase (or decrease) of activity in region B. Such an observation may reflect a relationship which is neurophysiologically interesting (e.g., activity in region $A$ causes activity in region $B$ ). Alternatively, this functional connectivity between $A$ and $B$ may simply be a downstream reflection of a common influence on areas $A$ and $B$ but not of any causal relationship between the two areas themselves (that is, activity in area $C$ causes activity in area $A$ and, separately, in area $B)$. Areas A and B may be considered effectively connected if their relationship can be shown to be a causal one. Effective connectivity is neurophysiologically more interesting than functional connectivity insofar as it deals with causal or influential relationships between brain regions.

It can be inferred from the descriptions of functional and effective connectivity that criteria for identifying them differ. Functional connectivity may be identified simply through observing how activity in two regions correlates over time. Effective connectivity requires an hypothesis-led approach in which an anatomical model, consisting of 2 or more regions, is defined. Connections between these regions are designated on the basis of empirical neuroanatomy and the connections are allocated weightings or path strengths in such a way that the model of inter-regional influences accounts best for the observed variance-covariance structure generated by the functional neuroimaging time series (Friston et al., 1993). Thus standard measures of effective connec- 
tivity or causal relationships between brain regions require a model which embodies an a priori hypothesis about which regions and which (directionally specified) connections are important in accounting for the data. A simplified evaluation of effective connectivity has recently been suggested by F riston et al. 1997). I t involves an assessment of the measured activity in a selected brain region in terms of the extent to which activity in that region predicts activity in other regions. Using a regression analysis with the activity from a voxel in the region of interest as the explanatory variable, and activity in every other voxel as response variables, one can identify those voxels in which there is a significant regression of the latter on the former. A significant regression may be interpreted as the existence of a coupling of activity between the explanatory and the response regions and has been conceptualized as evidence that activity in the former region "contributes" to activity in the latter (Friston et al., 1997). A significant regression of activity in area $\mathrm{C}$ on the product of activities in areas $A$ and $B$ similarly reflects an activitydependent contribution (from $A$ to $C$ modulated by $B$ or from $B$ to $C$ modulated by $A$ ). I t is this modulatory effect we examine below in groups of healthy controls and subjects with schizophrenia.

The hypothesis which drove the current analysis is motivated by three empirical observations. First, our previous studies suggested that there is an abnormal pattern of fronto-temporal interaction in schizophrenia. This has been manifest as a failure of the commonly observed deactivations of superior temporal cortex in the presence of prefrontal activation (Frith et al., 1995; Fletcher et al., 1996). It has also been explored in terms of connectivity and encapsulated as a disintegration of fronto-temporal connectivity (F riston and Frith, 1995). A second observation driving the analysis is the failure of anterior cingulate cortex activation in untreated schizophrenic subjects in association with performance of a modified verbal fluency task (Dolan et al., 1995). The third observation lies in the twofold effect of a dopaminergic perturbation on task-related activations in schizophrenic subjects. The dopaminergic perturbation is associated with an amelioration of the cingulate cortex hypoactivation and a trend toward normalization of thefronto-temporal relationship. This led to an explicit suggestion that "in schizophrenia, there is a dysregulation in the dopaminergic modulation of cingulate neuronal activity with a consequent impairment in the functional integration of more remote, but anatomically connected, cortical regions" (Dolan et al., 1995). The current analysis was therefore based upon the hypothesis that, in schizophrenia, there is an abnormality in the way in which theleft prefrontal cortex influences left superior temporal cortex and, further, that this abnormality is due, at least in part, to a failure of the anterior cingulate cortex to modulate the prefronto-temporal interactions.

\section{MATERIALS AND METHODS}

Twelve male subjects with DSM III-R diagnoses of schizophrenia and seven (six male; one female) agematched control subjects were studied. Subjects were free of neurological illness and had normal structural magnetic resonance imaging (MRI) scans. Throughout each PET scan, subjects engaged in learning and recall of word lists. Words were spoken at a rate of one per $2 \mathrm{~s}$. Retrieval was paced by the experimenter counting at the same rate. Learning and retrieval of the same list went on throughout a given 165-s scan. Across the 12 scans, lists varied in length from 1 to 12 items and the order was pseudo-randomized across subjects. So, for example, during the first scan, a subject might repeatedly hear and retrieve a 7-item word list, during the second scan a 3-item list, during the third a 12-item list and so on. Performance for each list presentation was recorded and both groups showed perfect recall with word lists of up to four words. Beyond this, performance declined most markedly in the schizophrenic subjects.

The analysis was designed to test our a priori model of abnormal cingulo-frontal modulation of temporal activity in schizophrenia. In terms of the PET observations, this abnormal neurophysiological relationship can be stated as follows: The normal pattern of cingulate modulation of prefronto-temporal interactions manifests as temporal cortical activity that is predicted by an interaction between activity in cingulate and prefrontal cortices. Thus, the prediction is that there will be a significant regression of temporal activity on the product of anterior cingulate and frontal activity. That is, the extent to which there will be coupling of activity between prefrontal and temporal cortices varies as a function of cingulate activity. We postulate that this relationship is abnormal in schizophrenia and used the PET data from the experiment described above (Fletcher et al., 1998) to test this hypothesis. Smoothed, spatially normalized, realigned images were analyzed using the general linear model. Activity in each voxel across the 12 scans was modeled as follows:

$$
\begin{aligned}
Y_{(\text {scans 1-12) }}=(P F C \times & \text { ACC })_{\text {scans 1-12 }} \\
& \times \beta+\left(P F C \gamma_{1}\right)+\left(A C C \gamma_{2}\right)+\text { Error, }
\end{aligned}
$$

where $Y$ is activity in region at each other voxel; $P F C$ is activity measured in prefrontal cortex; ACC is activity measured in anterior cingulate cortex; $\beta$ is Interactive contribution of PFC and ACC to region $Y$; and $\gamma_{1}$ and $\gamma_{2}$ are direct contributions from PFC and ACC respectively).

This is a simple regression equation in which the regression coefficient $\beta$ can be considered a measure of 
the way in which the product of PFC and ACC activity contributes to activity in any other voxel. The contribution of their combined activity to a given region will be considered significant if the regression coefficient accounts for a high proportion of variance in that region (compared to the error term). There are two other features of such an analysis worth noting. First, all of the variance in a given voxel that cannot be accounted for by a contribution from ACC and PFC is subsumed under the error term. This is an important difference from structural equation modeling in which all regions considered to be of importance are explicitly modelled. This means that a statement of contribution has less causal explanatory power than a statement of effective connectivity. Second, in the current analysis, we are concerned with the interactive effect of PFC and ACC on other brain regions. Thus, to ensure that we characterize only those effects predicted by the interaction between PFC and ACC activity, wetreated the effects of these two regions separately as confounds.

In view of our anatomically restricted hypothesis, we set a threshold in superior temporal cortex at $\mathrm{P}<0.05$ uncorrected for multiple comparisons.

\section{RESULTS}

The results are summarized in Fig. 1. In control subjects the product of PFC and ACC activity significantly predicted a superior temporal decrease. This was not the case in the schizophrenic group. A direct contrast between the two groups showed a significant difference between them in this respect.

\section{DISCUSSION}

The majority of neurological research in schizophrenia has been predicated on a segregationist perspective. However, one important historical view of the pathophysiology of the psychoses has emphasized that a disorder of integrative function could provide the mechanistic basis for the observed psychological dysfunction (Wernicke, 1874). A splitting or disintegration of psychological function was emphasized in the earliest phenomenological formulations of schizophrenia and the concept of a split is, of course, inherent in the name itself (Bleuler, 1987). Previous functional neuroimaging data have suggested that connectivity may indeed be disrupted in schizophrenic people (Friston and Frith, 1995). This abnormality is thought to lie in the integration of fronto-temporal function. The findings described above are consistent with this position and, further, suggest that one abnormal interaction may be underpinned by a failure of normal cingulate cortical modulatory control. This, too, is consistent with previous findings, suggesting that dopaminergic rever- sal of a cingulate abnormality is associated with a relative normalization of the fronto-temporal relationship in untreated schizophrenic patients (Dolan et al., 1995). F urther, a more recent study exploring memory retrieval in schizophrenia points to an abnormal relationship between prefrontal and medial temporal cortical activity in association with the condition (Heckers et al., 1998).

While the relatively small numbers of subjects and the subtle effects described mean that the current report should be treated as preliminary, the hypothesis that schizophrenic patients show an abnormality of the cingulo-fronto-temporal relationship is strongly supported by our data. How, then, do we interpret this abnormality? Clearly, the superior temporal cortex in heal thy subjects is sensitive to a combination of activity in ACC and PFC above and beyond its sensitivity to activity of either region in isolation. Within the framework of our hypothesis, we suggest that this phenomenon reflects a modulatory effect of anterior cingulate cortex on prefrontal interaction with superior temporal cortex. Thus, there is a tendency for an increase in prefrontal cortical activity to supress superior temporal cortex. Our results show that this effect, in healthy subjects, is more pronounced in the face of high anterior cingulate cortical activity. In the schizophrenic subjects, this modulatory effect is not seen. We interpret this as indicating a failure of cingulate modulation of PFC influence on superior temporal cortex. Of course an alternative interpretation is that the prefrontal cortex is modulating the effect of anterior cingulate on superior temporal cortex. The data do not distinguish between these two possibilities but our hypothesis favors the former and the experimental question was framed with this formulation in mind.

Anatomically, it has been shown that anterior cingulate cortex has strong reciprocal connections with prefrontal (Petrides and Pandya, 1984; Goldman-Rakic, 1988) and with superior temporal cortex (Pandya et al., 1981). Further, functional neuroimaging studies have implicated it in a variety of cognitive tasks, including internal generation (Frith et al., 1991), selective and divided attention (Corbetta et al., 1991), and response inhibition in the context of theStroop task (Bench et al., 1993). More recently, using event-related functional $\mathrm{MRI}$, it has been shown that anterior cingulate cortex is sensitive to both increasing difficulty and erroneous responses in a continuous performance task, suggesting a role in performance monitoring (Carter et al., 1998). Of course, such descriptions are based upon ideas of functional segregation, the notion of a specific region having a specific function. However, we feel that the notions of such attentional and monitoring processes lend themselves to the integrationist perspective. It seems highly likely that attention represents a 


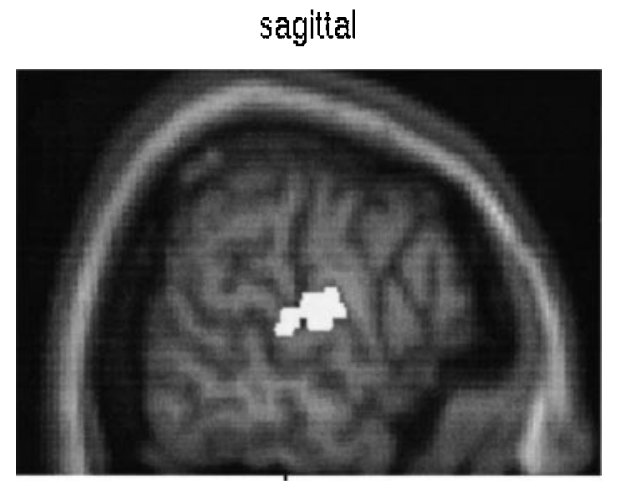

transverse

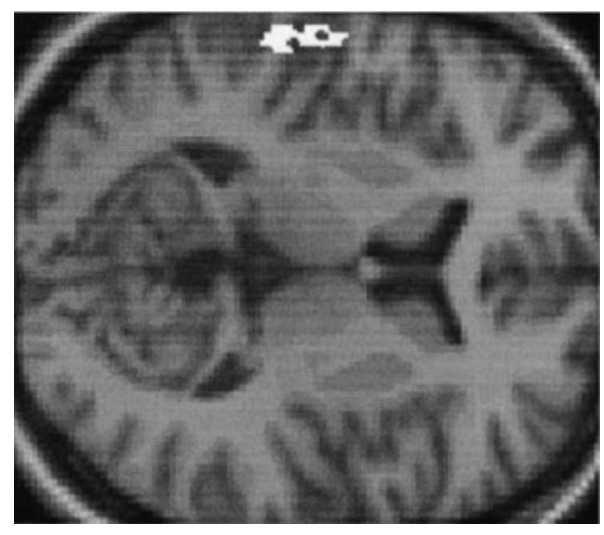

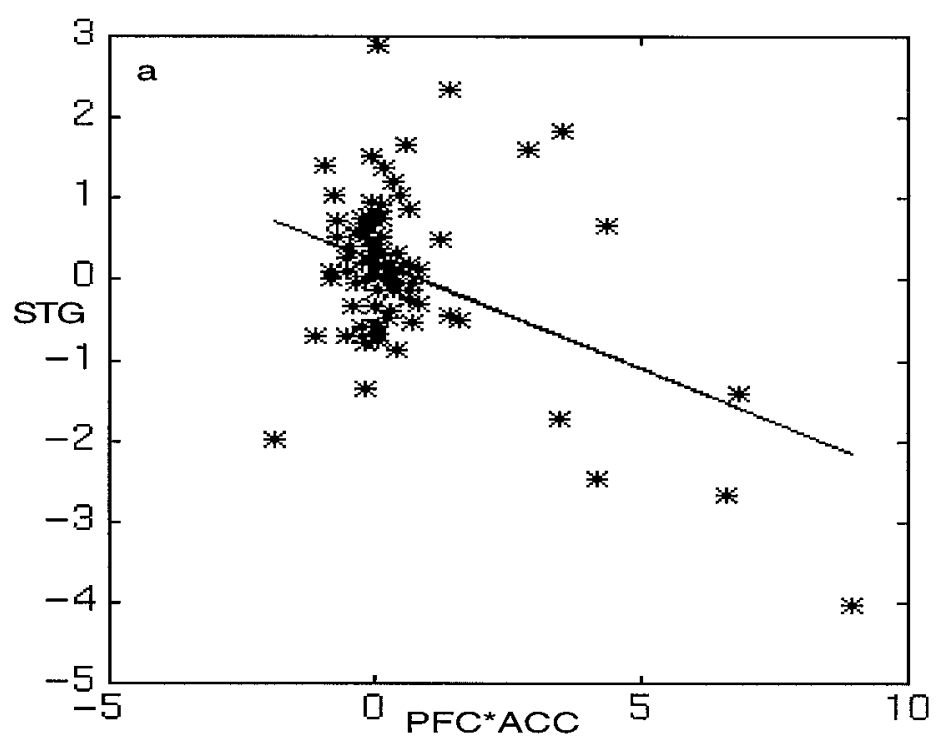

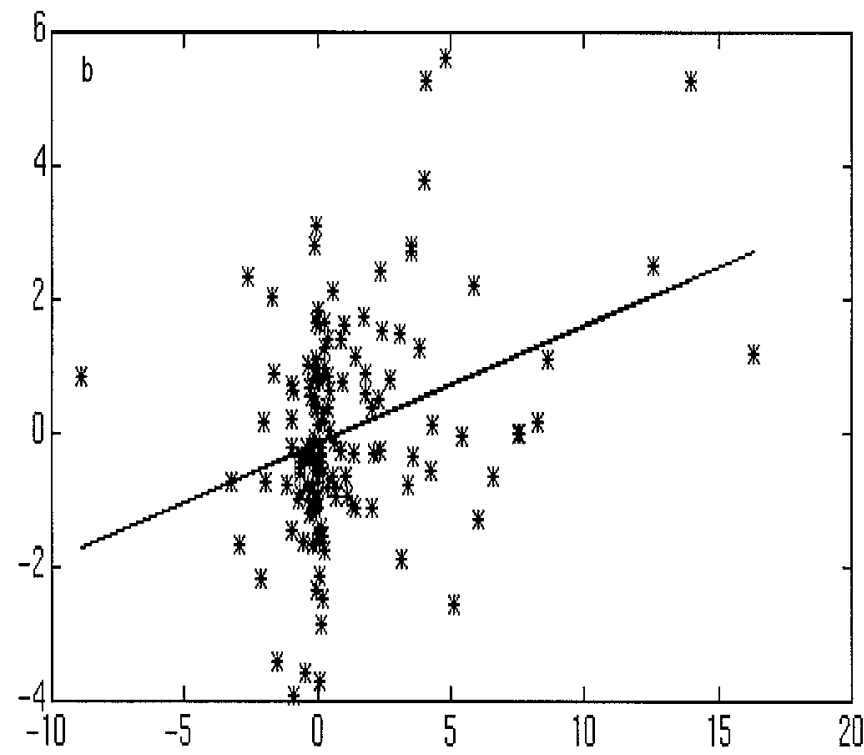

FIG. 1. Left panel: Region of left superior temporal cortex showing a significant difference between control and schizophrenic subjects $(\mathrm{P}<0.05)$. A statistical parametric map rendered onto saggital and transverse section of a stereotactically normalised structural mri is shown. Right panel: Graphic representation of the relationship between activity in left superior temporal cortex (y axis) and the combination of cingulo-prefrontal activity (x axis) in (a) controls and (b) schizophrenic subjects. The linear best fit is shown for both groups. The regression coefficient of each of the two groups is considered to provide a measure of Contribution of ACC $\times$ PFC to superior temporal cortical activity. Thus, in the controls an increase in PFC $\times$ ACC produces an inhibition of superior temporal activity. In the schizophrenic subjects the line slopes upward, indicating the opposite effect.

modulation of regional brain activity and, as such, is perhaps best conceptualized in terms of modulatory interactions between brain regions. The current model characterizes our data in just this way. Also, pertinent to the present findings, is a study of verbal memory encoding (Fletcher et al., 1995) in which it was found that a concurrently performed distraction task produced an attenuation of prefrontal activation in conjunction with augmentation of anterior cingulate activation. The observed pattern of a reduction in PFC activation and an increase in cingulate activation was thought to be compatible with the latter exerting a modulatory effect on neuronal activity in the former.

This notion of the cingulate cortex exerting modulatory effects on other regions and the disruption of this function in schizophrenia gains support from our hypothesis-directed analysis and complements our previous study (Dolan et al., 1995; Fletcher et al., 1996) where a failure of task-associated cingulate activation was associated with an abnormal fronto-temporal rela- 
tionship, a phenomenon which was ameliorated when a dopaminergic perturbation resulted in increased cingulate activation in the schizophrenic subjects. That schizophrenia may be a disorder of inter-regional connections has been suggested by observations that psychotic, schizophrenia-like symptoms are a feature of metachromatic leukodystrophy, a disease that includes demyelination of subfrontal white matter (Hyde et al., 1992; F riston and Frith, 1995).

Our data, therefore, imply that the anterior cingulate cortex may be a key region in the functional characterization of schizophrenia. This is in keeping with previous functional neuroimaging studies (Andreasen et al., 1992; Dolan et al., 1995) as well as neurophysiological data, suggesting schizophrenia-related abnormalities in anterior cingulate cortex (Benes et al., 1987, 1992). The suggested roles for this region in attention (Corbetta et al., 1991; Bench et al., 1993) and performance monitoring (Carter et al., 1998) are compatible with the pattern of cognitive deficits in attention (McGhie and Chapman, 1961) and working memory (Park and Holzman, 1992) reported in association with the disease. Additionally, recent functional neuroimaging of healthy controls suggesting a role for the region in performance monitoring (Carter et al., 1998) is compatible with the suggestion that a core cognitive deficit in schizophrenia lies in the failure to monitor internally generated actions (F rith, 1992). In our graded memory task, the normal reciprocal connectivity between prefrontal and temporal cortex, modulated by cingulate cortex, may subserve this monitoring function and is disrupted in the patient group. It is noteworthy, therefore, that a disturbance in monitoring has been thought tolieat the root of delusions and hallucinations that are characteristic of schizophrenia (Frith, 1992).

To summarize, this paper draws attention to an important approach to the description of functional neuroi maging data. Such an approach enables a characterization of normal and abnormal functional neuroanatomy in terms of integration between brain regions, a perspective which, we believe, more accurately captures the nature of the functional deficits in schizophrenia.

\section{REFERENCES}

Andreasen, N. C., Rezai, K., Alliger, R., Swayze, V. W., Flaum, M., Kirchner, P., Cohen, G., and O'Leary, D. S. 1992. Hypofrontality in neuroleptic-naive patients and in patients with chronic schizophrenia: Assessment with Xenon 133 single-photon emission computed tomography and the Tower of London. Arch. Gen. Psychiatry 49:943-958.

Andreasen, N. C. 1996. Pieces of the schizophrenia puzzle fall into place. Neuron 16:697-700.

Bench, C. J ., Frith, C. D., Grasby, P. M., Friston, K. J ., Paulesu, E., Frackowiak, R. S. J ., and Dolan, R. J . 1993. Investigations of the functional anatomy of attention using the Stroop test. Neuropsychologia 31:907-922.
Benes, F. M., Majocha, R., Bird, E. D., and Marotta, C. A. 1987. Increased Vertical Axon Numbers in Cingulate Cortex of Schizophrenics. Arch. Gen. Psychiatry 44:1017-1021.

Benes, F. M., Vincent, S. I., Alsterberg, G., Bird, E. D., and SanGiovanni, J . P. 1992. Increased GABA G $_{a}$ receptor binding in superficial layers of schizophrenic cingulate cortex. J . Neurosci. 12:924-929.

Bleuler, E. 1987. Dementia Praeco or the group of schizophrenias. In The Clinical Routes of the Schizophrenia Concept (J . Cutting and M. Shepherd, Eds.) Cambridge Univ. Press, Cambridge.

Carter, C. S., Braver, T. S., Barch, D. M., Botvinick, M. M., Noll, D., and Cohen, J. D. 1998. Anterior cingulate cortex, error detection and the online monitoring of performance. Science 280:747-749.

Chua, S. E., and McKenna, P.J . 1995. Schizophrenia, a brain disease? A critical review of structural and functional review of cerebral abnormality in the disorder. Br. J . Psychiatry 166:563-582.

Corbetta, M., Miezin, F. M., Dobmeyer, S., Shulman, G. L., and Petersen, S. E. 1991. Selective and divided attention during visual discriminations of shape, colour, and speed: F unctional anatomy by Positron Emission Tomography. J . Neurosci. 11(8):2383-2402.

Dolan, R. J ., Fletcher, P., Frith, C. D., Friston, K. J ., Frackowiak, R. S. J ., and Grasby, P. J . 1995. Dopaminergic modulation of an impaired cognitive activation in the anterior cingulate cortex in schizophrenia. Nature 378:180-182.

Fletcher, P. C., Frith, C. D., Grasby, P. M., Shallice, T., Frackowiak, R. S. J ., and Dolan, R. J . 1995. Brain systems for encoding and retrieval of auditory-verbal memory: An in vivo study in humans. Brain 118:401-416.

Fletcher, P. C., Frith, C. D., Grasby, P. M., Friston, K. J ., and Dolan, R. J. 1996. Local and distributed effects of apomorphine on fronto-temporal function in acute unmedicated schizophrenia. J . Neurosci. 16(21):7055-7062.

Fletcher, P. C., McKenna, P. J ., Frith, C. D., Grasby, P. M., Friston, K. J ., and Dolan, R. J . 1998. Brain activations in schizophrenia during a graded memory task studied with functional neuroimaging. Arch. Gen. Psychiatry, in press.

Friston, K. J ., Frith, C. D., Liddle, P., and Frackowiak, R. S. J . 1993. Functional connectivity: The principal component analysis of large (PET) data sets. J . Cereb. Blood Flow Metab. 13:5-14.

Friston, K. J ., and Frith, C. D. 1995. Schizophrenia: A disconnection syndrome? Clin. Neurosci. 3:89-97.

Friston, K. J ., Büchel, C., Fink, G. R., Morris, J ., Rolls, E., and Dolan, R. J . 1997. Psychophysiological and modulatory interactions in neuroimaging. Neuroimage 6:218-229.

Friston, K. J ., Frith, C. D., and Frackowiak, R. S. J . 1993. Timedependent changes in effective connectivity measured with PET. Human Brain Mapping 1:69-79.

Frith, C. D., Friston, K. J ., Liddle, P. F., and Frackowiak, R. S. J . 1991. Willed action and the prefrontal cortex in man: A study with PET. Proc. R. Soc. Lond. B 244:241-246.

Frith, C. D. 1992. The Cognitive Neuropsychology of Schizophrenia. Lawrence Erlbaum, Hove.

Frith, C. D., Friston, K. J ., Herold, S., Silbersweig, D., Fletcher, P., Cahill, C., Dolan, R. J ., Frackowiak, R. S. J ., and Liddle, P. F. 1995. Regional brain activity in chronic schizophrenic patients during the performance of a verbal fluency task. Br. J . Psychiatry 167:343349.

Goldman-Rakic, P. S. 1988. Topography Of Cognition: Parallel Distributed Networks in PrimateAssociation Cortex. Annu. Rev. Neurosci. 11:137-156.

Heckers, S., Rauch, S. L., Goff, D., Savage, C. R., Schacter, D. L., Fischmann, A. J ., and Alpert, N. M. 1998. I mpaired recruitment of the hippocampus during conscious recollection in schizophrenia. Nat. Neurosci. 1(4):318-323. 
Hyde, T. M., Ziegler, J . C., and Weinberger, D. R. 1992. Psychiatric disturbances in metachromatic leukodystrophy: Insights into the neurobiology of psychosis. Arch. Neurol . 49:401-406.

McGhie, A., and Chapman, J. 1961. Disorders of attention and perception in early schizophrenia. Br. J . Med. Psychol 34:103-116.

Pandya, D. N., Van Hoesen, G. W., and Mesulam, M.-M. 1981. Efferent Connections of the Cingulate Gyrus in the Rhesus Monkey. Exp. Brain Res. 42:319-330.

Park, S., and Holzman, P. S. 1992. Schizophrenics show spatial working memory deficits. Arch. Gen. Psychiatry 49:975-982.
Petrides, M., and Pandya, D. N. 1984. Projections to the frontal cortex from the posterior parietal region in the rhesus monkey. J . Comp. Neurol. 228:105-116.

Phillips, C. G., Zeki, S., and Barlow, H. B. 1984. Localisation of function in the cerebral cortex. Past, present and future. Brain 107:327-361.

Tononi, G., Sporns, O., and Edelman, G. M. 1992. Reentry and the problem of integrating multiple cortical areas: Simulation of dynamic integration in the visual system. Cerebral Cortex 2:310-335.

Wernicke, C. 1874. Der Aphasiche Symptomenkomplex, Breslau. 